\title{
Cytokines induce tight junction disassembly in airway cells via an EGFR-dependent MAPK/ERK1/2-pathway
}

\author{
Loredana Petecchia ${ }^{1}$, Federica Sabatini ${ }^{1}$, Cesare Usai ${ }^{2}$, Emanuela Caci ${ }^{3}$, Luigi Varesio ${ }^{4}$ and Giovanni A Rossi ${ }^{1}$
}

Epithelial barrier permeability is altered in inflammatory respiratory disorders by a variety of noxious agents through modifications of the epithelial cell structure that possibly involve tight junction (TJ) organization. To evaluate in vitro whether pro-inflammatory cytokines involved in the pathogenesis of respiratory disorders could alter TJ organization and epithelial barrier integrity, and to characterize the signal transduction pathway involved Calu-3 airway epithelial cells were exposed to TNF- $\alpha$, IL-4 and IFN- $\gamma$ to assess changes in: (a) TJ assembly, that is, occludin and zonula occludens (ZO)-1 expression and localization, evaluated by confocal microscopy; (b) apoptotic activity, quantified using terminal transferase deoxyuridine triphosphate nick-end labeling staining; (c) epithelial barrier integrity, detected as transmembrane electrical resistance and expressed as $\mathrm{G}_{\mathrm{T}}$ values; (d) epidermal growth factor receptor (EGFR)-dependent mitogenactivated protein (MAP) kinase (MAPK)/extracellular signal-regulated kinases (ERK)1/2 phosphorylation, assessed by western blotting. Exposure to cytokines for $48 \mathrm{~h}$ induced a noticeable downregulation of the TJ transmembrane proteins. The degree ZO- 1 and occludin colocalization was $62 \pm 2 \%$ in control cultures and significantly decreased in the presence of TNF- $\alpha(47 \pm 3 \%)$, IL-4 (43 $\pm 1 \%)$ and INF- $\gamma(35 \pm 3 \%)$. Although no apoptosis induction was detected following exposure to cytokines, changes in the epithelial barrier integrity were observed, with a significant enhancement in paracellular conductance. $G_{T}$ values were, respectively, $1.030 \pm 0.0,1.300 \pm 0.04,1.260 \pm 0.020$ and $2.220 \pm 0.015\left(\mathrm{mS} / \mathrm{cm}^{2}\right) \times 1000$ in control cultures and in those exposed to TNF- $\alpha$, IFN- $\gamma$ and IL-4. The involvement of EGFR-dependent MAPK/ERK1/2 signaling pathway in cytokine-induced damage was demonstrated by a significant increase in threonine/tyrosine phosphorylation of ERK $1 / 2$, already detectable after 5 min incubation. All these cytokine-induced changes were markedly prevented when Calu-3 cells were cultured in the presence of an EGFR inhibitor (AG1478, $1 \mu \mathrm{M}$ ) or a MAP kinase inhibitor (U0126, $25 \mu \mathrm{M}$ ). In conclusion, cytokine-induced epithelial injury includes TJ disassembly and epithelial barrier permeability alteration and involves the EGFR-dependent MAPK/ERK1/2 signaling pathway.

Laboratory Investigation (2012) 92, 1140-1148; doi:10.1038/labinvest.2012.67; published online 14 May 2012

KEYWORDS: airway inflammation; IL-4; INF- $\gamma$; occludin; TNF- $\alpha$; zonula occludens family

One of the major functions of the airway epithelium is to act as a selective barrier, maintaining the integrity of tissue compartments and impeding entry of inhaled microorganisms, irritants and allergens. ${ }^{1}$ Epithelial barrier integrity is maintained by adhesive interactions occurring at the cell-cell and cell-matrix contacts by junctional proteins and focal adhesion complexes that are anchored to the cytoskeleton. ${ }^{2}$ This intercellular junctional complex includes tight junctions (TJs), adherens junctions, desmosomes and gap junctions. ${ }^{2,3}$ TJs are organized by the interactions between the transmembrane proteins (occludin, claudins and junction adhesion molecule) and the intracellular plaque zonula occludens (ZO)-1, ZO-2, ZO-3, cingulin, 7H6 and others and the actin cytoskeleton. ${ }^{4-7}$ The plaque protein $\mathrm{ZO}-1$ is shown to have a unique role in the formation of TJs during epithelial cellular polarization. At the initial stage of cell-cell contact of epithelial cells, primordial spot-like junctions are formed at the tips of thin cellular protrusions radiating from adjacent cells, where ZO-1 are precisely concentrated. In epithelial cells, ${ }^{8}$ but also in mesenchymal cells, ${ }^{9,10}$ occludin is accumulated at the ZO-1-positive spot-like junctions to form continuous TJ during the configuration of cell polarity.

${ }^{1}$ Pulmonary Disease Unit, G. Gaslini Institute, Genoa, Italy; ${ }^{2}$ Institute of Biophysics, National Research Council, Genoa, Italy; ${ }^{3}$ Laboratorio di Genetica Molecolare G. Gaslini Institute, Genoa, Italy and ${ }^{4}$ Molecular Biology Laboratory G. Gaslini Institute, Genoa, Italy

Correspondence: Dr L Petecchia, PhD, Pulmonary Diseases Unit G. Gaslini Institute, Largo G. Gaslini 5, 16147 Genoa, Italy.

E-mail: loredanapetecchia@gmail.com

Received 29 August 2011; revised 17 February 2012; accepted 27 February 2012 
A significant body of evidence indicates that TJs are associated with numerous intracellular signaling molecules, and are regulated by the activity of signal transduction pathways. ${ }^{4}$ Pro-inflammatory cytokines have been found to negatively affect epithelial barrier functions in vitro in experiments mainly conducted on the intestinal epithelial cell lines. Although epithelial damage and increased epithelial barrier permeability is also common in inflammatory conditions of the airways, ${ }^{11-13}$ a limited information is available on the airway epithelial cells. ${ }^{3}$

Several mechanisms can be responsible for the cytokineinduced disruption of epithelial barriers, including expressional downregulation of junctional proteins. A variety of mediators possess the capability to alter TJ structure and barrier function on a variety of epithelial cell types, ${ }^{11,14,15}$ but few studies have been performed on the bronchial epithelial cells, ${ }^{16-23}$ exploring the signaling pathways involved. ${ }^{19,21}$ Specifically, it has never been evaluated the possible engagement of the epidermal growth factor receptor (EGFR) signaling pathways, known to promote various cellular processes by phosphorylation of mitogen-activated protein (MAP) kinases. ${ }^{24}$ Activation of the MAP kinases-extracellular signalregulated kinases ${ }^{25}$ (MAPK/ERK)1/2 has been associated not only with cell survival, proliferation, growth and differentiation $^{24}$ but also with TJ protein disruption. ${ }^{12,26,27}$ A study was therefore designed to evaluate whether exposure to proinflammatory cytokines involved in the pathogenesis of infectious and allergic respiratory disorders could alter $\mathrm{TJ}$ and epithelial barrier integrity by dysregulation of ZO-1 and occludin and to define the possible involvement of EGFR/ MAPK/ERK1/2 cascade.

\section{MATERIALS AND METHODS Epithelial Cell Culture}

Calu- $3^{28}$ adenocarcinoma cells (a kind gift from Dr Diego Ferrone, University of Genova, Italy) were selected for these experiments because of their highly characterized intercellular adhesion molecules and ability to form monolayers that are highly impermeable to ionic and macromolecular flux. $^{29,30}$ Cells were grown in T-75 culture flasks in an atmosphere of $5 \% \mathrm{CO}_{2}$ at $37^{\circ} \mathrm{C}$, and used in passage numbers 20-30. For all experiments comparing different culture conditions, cells in similar passage numbers were used. The cells were maintained in a 1:1 mixture of DMEM/ Ham's F12 medium (Euroclone, Europe) supplemented with fetal bovine serum, non-essential amino acids and penicillin/ streptomycin (Euroclone, Europe). Cell viability was evaluated by trypan blue dye exclusion test (Euroclone).

\section{Occludin and ZO-1 Expression and Localization}

To evaluate TJs (occludin and ZO-1) expression and localization, Calu-3 cells were grown to confluence on coverslips and incubated for $48 \mathrm{~h}$ with TNF- $\alpha(10 \mathrm{ng} / \mathrm{ml}),{ }^{31} \mathrm{IL}-4(5 \mathrm{ng} /$ $\mathrm{ml})^{17}$ and IFN- $\gamma(50 \mathrm{ng} / \mathrm{ml})^{17}$ (Invitrogen, Milano, Italy). At these possibly clinically relevant doses, ${ }^{18}$ these cytokines were reported to induce barrier breakdown in a rapid, dose-dependent fashion in Calu-3. ${ }^{16-19,31}$ Preliminary experiments in our lab confirmed the results reported by others. Cells were then permeabilized and stained as previously described. ${ }^{4,12}$

After blocking, the preparations were incubated with mouse anti-human occludin and anti-rabbit human ZO-1 (Zymed Laboratories San Francisco, CA, USA), washed and incubated with Alexa-fluor 488 (green) goat antimouse and Alexa-fluor 647 (red) goat antirabbit (Invitrogen SRL, Italy).

For the negative control, normal mouse (for occludin) or rabbit (for ZO-1) non-specific immunoglobulins (Santa Cruz Biotechnology, Santa Cruz, CA, USA) were used at the same protein concentration as the primary antibody. Both green and red fluorescence were analyzed by confocal laser scanning microscopy (TCS SL microscope; Leica; Mannheim, Germany). Images were collected at the height of the layer where TJs are known to be expected. ${ }^{17}$

The quantitative estimation of colocalized proteins in immunocytochemical studies has been performed calculating the colocalization coefficients ${ }^{32}$ from the red- and greenchannel scatterplot.

Colocalization coefficients express the fraction of colocalizing molecular species in each component of a dual-colour image and are based on the Pearson's correlation coefficient, a standard procedure for matching one image with another in pattern recognition. ${ }^{33}$ If two molecular species are colocalized, the overlay of their spatial distributions has a correlation value higher than what would be expected by chance alone. Costes et al, ${ }^{34}$ developed an automated procedure to evaluate correlation between the green and red channels with a significance level $>95 \%$. The same procedure automatically determines an intensity threshold for each colour channel based on a linear least-square fit of the green and red intensities in the image's two-dimensional correlation cytofluorogram. Costes ${ }^{34}$ approach has been accomplished by macro routines integrated as plugins (WCIF Colocalization Plugins, Wright Cell Imaging Facility, Toronto Western Research Institute, Canada) in the ImageJ $1.45 \mathrm{~g}$ software (Wayne Rasband, NIH, USA).

\section{Determination of DNA Damage}

Briefly, DNA damage was evaluated using terminal transferase deoxyuridine triphosphate nick-end labeling (TUNEL) staining following manufacturer's instructions (Roche Diagnostics GmbH, Germany), Briefly, Calu-3 cells $\left(5 \times 10^{-4}\right.$ per well) were plated in 24-well till subconfluency, and then exposed for $48 \mathrm{~h}$ to TNF- $\alpha(10 \mathrm{ng} / \mathrm{ml})$, IL- $4(5 \mathrm{ng} / \mathrm{ml})$ and IFN- $\gamma(50 \mathrm{ng} / \mathrm{ml})$.

As apoptotic cells are extruded from epithelia 'anoikis' fairly rapidly ${ }^{35} 48 \mathrm{~h}$ following cytokine exposure, the apical surfaces of epithelia were washed to collect extruded cells while Calu-3 cells were trypsinized. The PBS-washed and -trypsinized cells were then loaded into a cytospin apparatus, cytocentrifuged onto polysine glass slides, fixed in 
$4 \%$ paraformaldehyde in PBS for $1 \mathrm{~h}$ at room temperature and washed with PBS. Cells were then permeabilized and labeled as previously described. ${ }^{12}$ Rinsed slides were then coverslipped with a mounting medium containing $4^{\prime}, 6^{\prime}$-diamidino-2-phenylindole (DAPI; Vector Laboratories, Burlingame, CA, USA) for nuclear counterstaining. TUNEL ${ }^{+}$ apoptotic cells, which fluoresce bright green, were viewed with a Nikon Eclipse E1000 fluorescent microscope (Nikon, Tokyo) equipped with a FITC filter. To avoid erroneously false-positive results, Calu-3 cells treated with DNase I were used as a positive control.

\section{Measurement of Transepithelial Electrical Resistance (TER)}

To assess epithelial barrier integrity, Calu-3 were plated $\left(0.5 \times 10^{6}\right.$ cells per well $)$ and cultured for 5 days and then exposed to TNF- $\alpha(10 \mathrm{ng} / \mathrm{ml})$, IL-4 $(5 \mathrm{ng} / \mathrm{ml})$ and IFN- $\gamma$ $(50 \mathrm{ng} / \mathrm{ml})$ for $48 \mathrm{~h}$. As a positive control, the $\mathrm{Ca}^{2+}$ chelator EDTA, $1 \mathrm{mM}$, was used to increase cell permeability and added to the apical site of the Calu-3 cell cultures for $2 \mathrm{~h} .{ }^{36}$ TER $\left(\Omega . \mathrm{cm}^{2}\right)$ or total conductance $\left(G_{T}\right)$ were measured with an epithelial voltmeter (Millipore-ERS, Millipore) using chopstick-like electrodes as a measurement of tight junctional barrier formation. For experiments, cells were seeded at high density on Snapwell inserts (Costar, Corning) and maintained at $37^{\circ} \mathrm{C}$ in a $5 \% \mathrm{CO} 2,95 \%$ air atmosphere, as previously described. ${ }^{37}$

TER values shown as $G_{T}\left(1 / \text { TER } \times 1000, \mathrm{mS} / \mathrm{cm}^{2}\right)^{37}$ were obtained by subtracting blank filter resistance $\left(30 \Omega . \mathrm{cm}^{2}\right)$ from all readings.

\section{Immunoblotting for Native and Activated ERK1/2}

Calu-3 cells $\left(0.5 \times 10^{6}\right.$ cells per well $)$ were cultured in Petri culture dishes to $90 \%$ confluence and then exposed to TNF- $\alpha$ $(10 \mathrm{ng} / \mathrm{ml}), \mathrm{IL}-4(5 \mathrm{ng} / \mathrm{ml})$ and IFN- $\gamma(50 \mathrm{ng} / \mathrm{ml})$ for $5 \mathrm{~min}$. The same amount of protein from each line $(50 \mu \mathrm{g})$ was separated by $10 \%$ SDS-PAGE, transferred to PVDF membranes. Membrane was blotted for rabbit anti-human total ERK1/2 (Tot-ERK1/2) and phosphorylated ERK1/2 (P-ERK1/2; Cell Signalling Technology, Beverly, MA, USA). ${ }^{17}$ After incubation with HPR-conjugated anti-rabbit antibody (Cell Signaling Technology), the bands were detected using enhanced chemoluminescence (ECL, Pierce, Celbio, Italy) and relevant band intensities were quantified using a Versadoc Imaging System model 3000 (Biorad Laboratories, Hercules, CA, USA).

\section{Involvement of EGFR/MAPK/ERK1/2 Pathway in Cytokine-Induced Changes}

To demonstrate the involvement of the EGFR-dependent MAPK/ERK1/2 signaling pathway, in some experiments, cells were pre-incubated for $1 \mathrm{~h}$ with the EGFR tyrosine kinase inhibitor AG1478 ( $1 \mu \mathrm{M}$; Calbiochem Inalco SpA; Milan, Italy) or the MAP kinase (MEK) inhibitor, U0126 $(25 \mu \mathrm{M}$; Sigma-Aldrich, St Louis, MO, USA) ${ }^{12}$ before being exposed to TNF- $\alpha$, IL-4 and IFN- $\gamma$.

\section{Statistical Analysis}

Statistical evaluation was performed using the statistical software package GraphPad Prism 3.02 (GraphPad Software, San Diego, CA, USA). The results from relevant band intensities and colocalization analysis were expressed as mean \pm s.e.m. and Paired $t$ test or Unpaired $t$ test were used. Probability values $(P<0.05)$ were considered as statistically significant.

\section{RESULTS}

\section{TJ Expression in Calu-3 Cells}

Calu-3 cells cultures showed a strong positivity for occludin (green fluorescence) (Figure 1a) and ZO-1 (red fluorescence) (Figure 1b) appearing as contiguous rings around the peripheral margins of the cells, colocalized at sites of cell-cell contact (Figure 1c). Figures 1d-f show the negative control. Exposure to TNF- $\alpha$, IL- 4 or INF- $\gamma$ for $48 \mathrm{~h}$ induced a noticeable downregulation of TJs, with a stronger suppression of ZO-1 than of occludin (Figures 1a-q) confirmed by quantitative analysis (Figure 2). The degree ZO-1 and occludin colocalization detected in control cultures $(62 \pm 2 \%)$ was decreased in the presence of TNF- $\alpha(47 \pm 3 \% ; P<0.05)$, IL-4 $(43 \pm 1 \% ; P<0.01)$ and INF- $\gamma(35 \pm 3 \% ; P<0.001)$, with a significant difference between the damage induced by INF- $\gamma$, as compared with TNF- $\alpha(P<0.01)$ and IL-4 $(P<0.05)$.

\section{Determination of DNA Damage by TUNEL Assay}

To evaluate whether the disassembly of TJs could be related to pro-apoptotic effects, modifications of cell viability were tested as DNA damage by TUNEL assay. As shown in Figure 3, only a very low number of cells exhibiting a faint fluorescent signal was detected in control cultures (Figure 3a). We treated Calu 3 cells with Dnase I as a positive control (Figure 3b). Neither significant morphological changes nor DNA damage was observed in Calu-3 after exposure to TNF- $\alpha$, IL-4 or IFN- $\gamma$ (Figures $3 \mathrm{c}-\mathrm{e}$ ).

\section{Transepithelial Electrical Resistance}

As expected, exposure to $\mathrm{Ca}^{2+}$ chelator EDTA for $2 \mathrm{~h}$ caused a significant increase in paracellular conductance, $G_{T}$ levels being $0.705 \pm 0.06\left(\mathrm{mS} / \mathrm{cm}^{2}\right) \times 1000$ in control cultures and $1.513 \pm 0.05\left(\mathrm{mS} / \mathrm{cm}^{2}\right) \times 1000$ in EDTA exposed cultures $(P<0.001)$ (Figure 4a). Similarly, also exposure to TNF- $\alpha$, IL-4 and IFN- $\gamma$ for $48 \mathrm{~h}$ resulted in a detectable reduction in barrier function, associated with an enhancement in paracellular conductance (Figure $4 \mathrm{~b}$ ). $G_{T}$ values, $1.030 \pm 0.03$ $\left(\mathrm{mS} / \mathrm{cm}^{2}\right) \times 1000$ in control cultures, were increased at similar levels after exposure to TNF- $\alpha$ and IFN- $\gamma$ $\left(1.300 \pm 0.04 \quad\left(\mathrm{mS} / \mathrm{cm}^{2}\right) \times 1000\right.$ and $1.260 \pm 0.020 \quad(\mathrm{mS} /$ $\left.\mathrm{cm}^{2}\right) \times 1000$, respectively; $P<0.0001$, each comparison), and even more in cells exposed to IL-4 $(2.220 \pm 0.015(\mathrm{mS} /$ $\left.\left.\mathrm{cm}^{2}\right) \times 1000 ; P<0.001\right)$. 

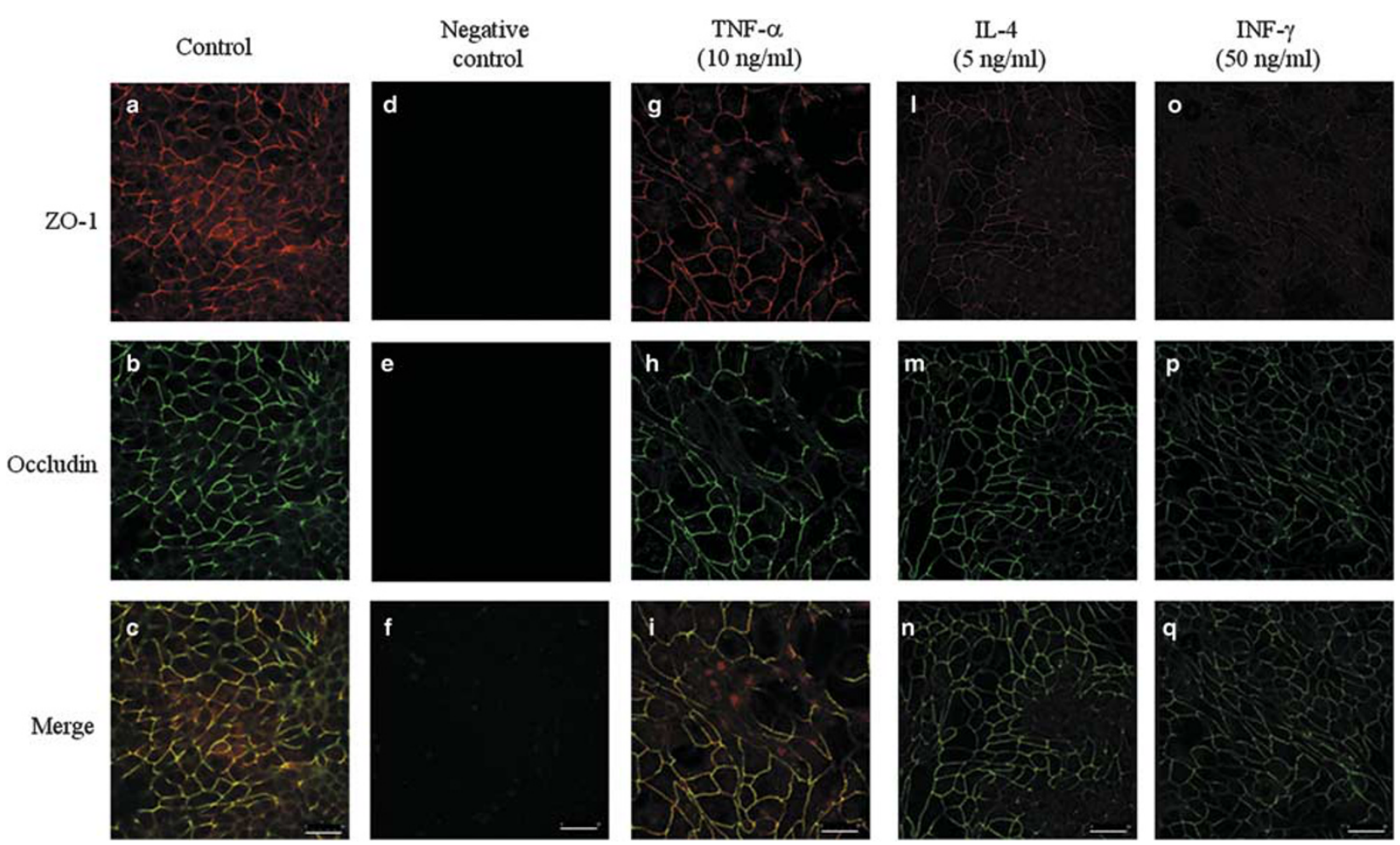

Figure 1 Representative photomicrographs obtained by fluorescent microscopy after immunofluorescence staining of zonula occludens (ZO)-1 and occludin expression in Calu-3 cells under basal culture conditions (a-f) or after stimulation for $48 \mathrm{~h}$ with TNF- $\alpha,(\mathbf{g}-\mathbf{i})$, IL-4 (I-n) or INF- $\gamma$ (o-q). Red staining (top panels) represents ZO-1, green staining (middle panels) occludin and (bottom panels) merged images of both occludin and ZO-1, where areas of colocalization appear as yellow. Unstimulated cells (a-c) stained with normal mouse (for occludin) or rabbit (for ZO-1) non-specific immunoglobulins were used as negative control. Original magnification $\times 63$. Each picture is representing one confocal layer. The results shown are representative of the results obtained in six independent experiments. Scale bar: $50 \mu \mathrm{m}$.

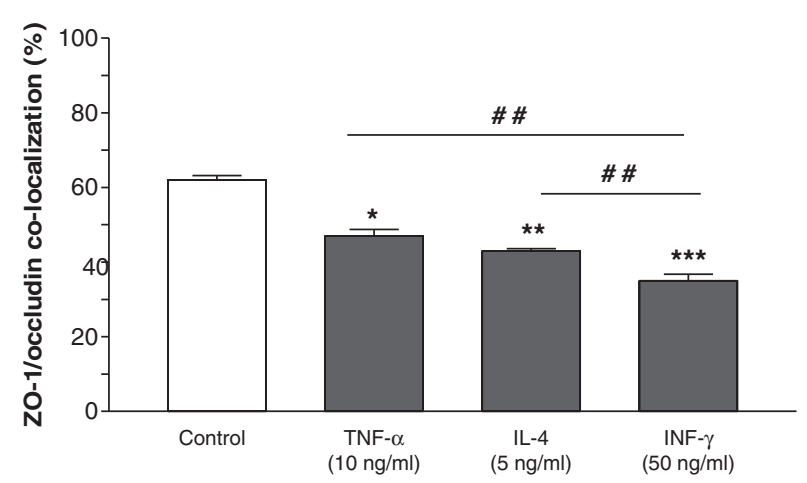

Figure 2 Quantitative evaluation of the percentage of zonula occludens (ZO)-1/occludin colocalization in Calu-3 cells under basal culture conditions (Control) or after exposure to TNF- $\alpha$, IL-4 or INF- $\gamma$. Data are reported as mean \pm s.e.m.. ${ }^{\star} P<0.05,{ }^{* * P}<0.01$ and ${ }^{* * *} P<0.001$, versus control; ${ }^{\# \#} P<0.01$ versus INF- $\gamma$-treated cells.

\section{ERK1/2 Pathway Activation}

To evaluate the possible involvement ERK1/2 pathway, Calu-3 cells were exposed to TNF- $\alpha$, IL- 4 and IFN- $\gamma$ for $5 \mathrm{~min}$. An increased threonine/tyrosine phosphorylation of ERK1/2 was already detectable by western blotting at $5 \mathrm{~min}$ (Figure 5a), with a detectable increase of the phosphorylated/
Tot-ERK1/2 ratio, as compared with unstimulated cultures. Quantitative analysis showed a similar increase of the phosphorylated/Tot-ERK1/2 ratio in cell cultures exposed to TNF$\alpha$, IL-4 and IFN- $\gamma(P<0.01$, each comparison) (Figure $5 b)$.

\section{Involvement of EGFR in Cytokine-Induced Activation of the ERK1/2 Pathway}

To determine whether EGFR is required for cytokine-induced ERK1/2 activation, Calu-3 cells were pre-incubated with AG1478 or with U0126. Pre-incubation of the cells for $1 \mathrm{~h}$ with AG1478 almost completely prevented the cytokine-induced ERK1/2 phosphorylation (Figure 6a) and the associated changes in the phosphorylated/Tot-ERK1/2 ratio. The quantitative analysis showed that the degree of inhibition was similar in cell cultures stimulated with TNF- $\alpha$, IL-4 and IFN$\gamma(P<0.001$, each comparison) (Figure 6b). Similar results were observed when the cells were pre-incubated with U0126 (Figure 6).

\section{Involvement of ERK1/2 Pathway Activation via EGFR- MEK in the Cytokine-Induced Damage}

Confocal microscopy showed that pre-exposure of Calu-3 cells to AG1478 or U0126 almost completely prevented ZO-1 and occludin junctional organization induced by TNF- $\alpha$ 
Control

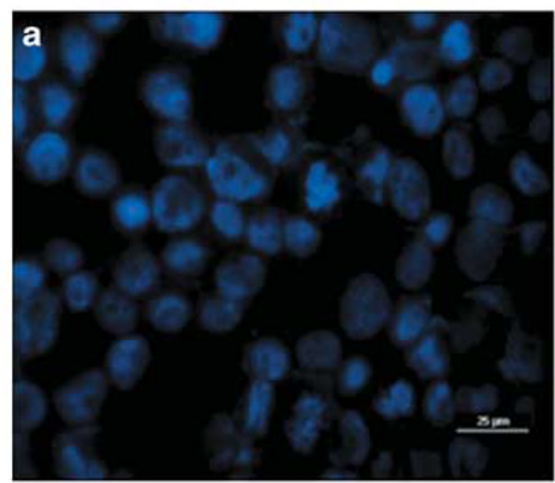

TNF- $\alpha(10 \mathrm{ng} / \mathrm{ml})$

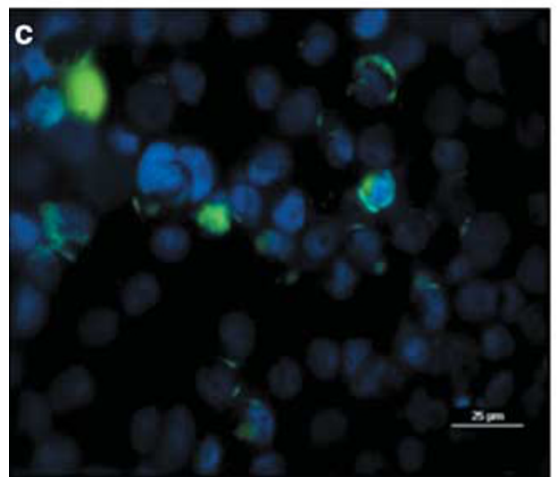

IL-4 (5 ng/ml)

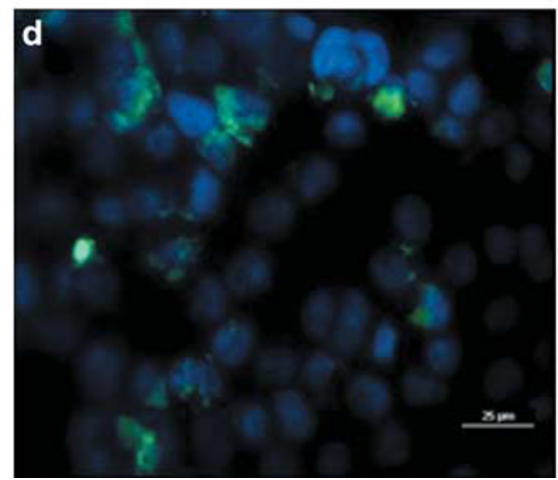

DNAse I

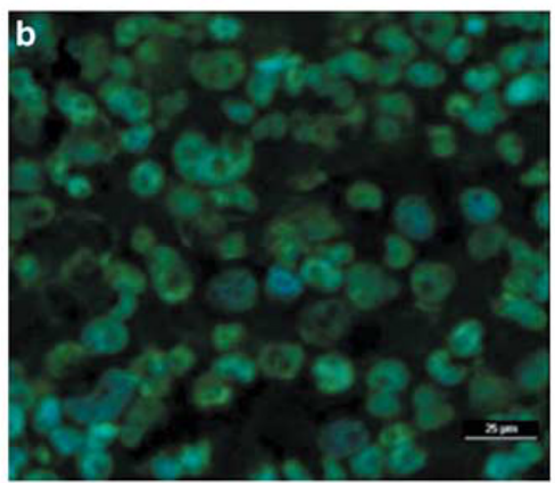

Figure 3 Representative photomicrographs obtained by fluorescent microscopy after immunofluorescence staining of the analysis of DNA cleavage by terminal transferase deoxyuridine triphosphate nick-end labeling (TUNEL) assay in Calu-3 cultured in medium alone (Control), (a) with DNAse I (positive control) (b) or in the presence of TNF- $\alpha$, (c) IL-4 (d) and INF- $\gamma$ (e) for $48 \mathrm{~h}$. Apoptotic nuclei are displayed by green fluorescence whereas viable DAPI counterstained nuclei appeared blue. Original magnification $\times 40$. The results shown are representative of five independent experiments. Scale bar: $25 \mu \mathrm{m}$.
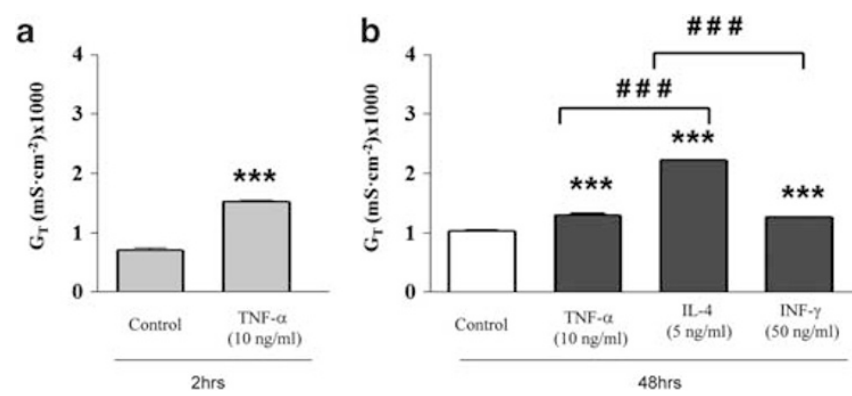

Figure 4 Quantitative evaluation of the effects of EDTA, TNF- $\alpha$, IL-4 and INF- $\gamma$ on transepithelial electrical resistance (TER) in Calu-3 cells under basal culture conditions (Control) or after stimulation for $2 \mathrm{~h}$ with EDTA (1 $\mathrm{mM}$ ) (a) and for $48 \mathrm{~h}$ TNF- $\alpha$, IL-4 and INF- $\gamma(\mathbf{b})$. Data are reported as mean \pm s.e., $(n=3)$. The results shown are representative of five independent experiments. ${ }^{* *} P<0.001$ versus control; ${ }^{\# \# \#} P<0.01$ versus IL-4-treated cells.

(Figures 7a-c), IL-4 (Figures 7d-f) and INF- $\gamma$ (Figures 7g-i). These modifications were confirmed by quantitative analysis, showing that both AG1478 and U0126 significantly and similarly inhibited the degree of TJ damage induced by TNF- $\alpha$ $(P<0.05)$, IL-4 $(P<0.01)$ and INF- $\gamma(P<0.05)$ (Figure 8). The addition of AG1478 or U0126 to confluent Calu-3 before cytokine exposure also completely restored the barrier dysfunction induced by TNF- $\alpha(P<0.05)$, IL-4 $(P<0.01)$ and INF- $\gamma(P<0.05)$ (Figure 9).

\section{DISCUSSION}

The present study shows that exposure to TNF- $\alpha$, IL-4 or IFN- $\gamma$ induced a remarkable reduction in the expression of transmembrane TJ components, occludin and ZO-1, associated with their spatial dislocation, through an EGFR-dependent MAPK/ERK1/2 signaling pathway. The disassembly of TJs observed was associated with modification of the epithelial barrier integrity leading to a decrease in TER. Cell apoptosis was not detected by TUNEL assay, suggesting that TJ disassembly may be an 'early event,' a sensitive indicator of initial bronchial epithelial cells injury, before DNA damage can be perceived. Apoptotic cells are extruded from epithelia 'anoikis' fairly rapidly. The quantification of DNA damage, or evaluation of apoptosis can be missed at any given time if the assays are done in the epithelia. ${ }^{35}$ Epithelial barrier integrity and functions are determined by lateral adhesive plasma membrane structures known as intercellular junctions, including ZO-1 and occludin, which constitute the TJs ${ }^{38} \mathrm{ZO}-1$ has a pivotal role in $\mathrm{TJ}$ formation and organization by linking 
a

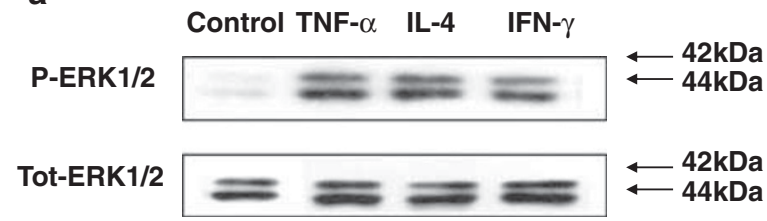

b

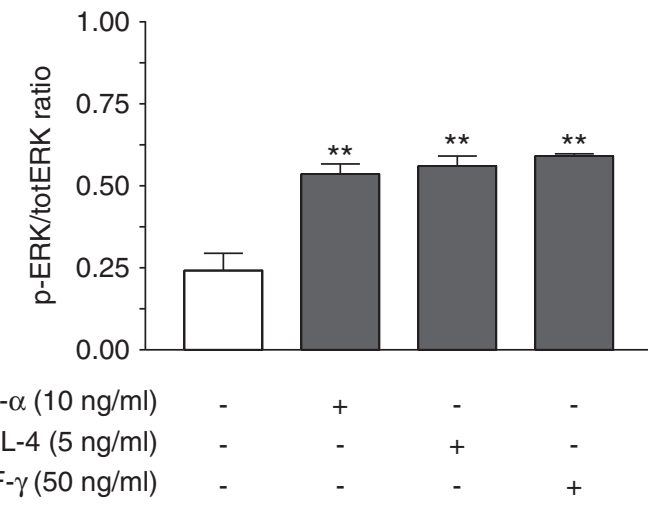

Figure 5 Cytokine-induced phosphorylation of extracellular signalregulated kinases (ERK)1/2. (a) Representative western blots for total (TotERK1/2) and phosphorylated ERK1/2 (P-ERK1/2) levels in Calu-3 cultured with medium alone (Control) or exposed to TNF- $\alpha$, IL-4 and INF- $\gamma$ for $5 \mathrm{~min}$. Arrows indicate the molecular size of the ERK1 (42 kDa), ERK2 (44 kDa).

(b) After densitometric analysis, P-ERK $1 / 2$ data were normalized to Tot-ERK $1 / 2$ and plotted as means \pm s.e.m.. The results shown are representative of five independent experiments. ${ }^{* *} P<0.01$ versus control.

the transmembrane protein occludin to other cytoplasmic components of the TJ complex and to the actin cytoskeleton. ${ }^{2}$ Occludin is an integral membrane protein localizing at TJ with four transmembrane domains and a long $\mathrm{COOH}$ terminal cytoplasmic domain, necessary for links to ZO-1 and the cytoskeleton. ${ }^{2}$

However, it has been shown that occludin is not required for the formation of TJ strands in the tissues such as the intestine, liver and kidney of knockout mice, but it is suggested that claudins, another transmembrane $\mathrm{TJ}$ protein, take over the role of occludin. ${ }^{39}$

TJs possess significant adhesive strength to maintain the integrity of epithelial layers and to form a tight barrier to paracellular flux of macromolecules, but these junctional complexes are also characterized by an intrinsic plasticity manifested as the ability to rapidly disassemble and reestablish their structures. ${ }^{40}$ This junctional plasticity is essential in normal condition, but if occurs at a higher rate, as in disease states, may result in TJ disassembly with breakdown of epithelial barrier integrity. ${ }^{41,42}$ Disruption of TJ structure and increased epithelial permeability, induced by pollutants, microbial products, oxidative agents, proteases and cytokines, is a common event in acute and chronic inflammatory conditions. ${ }^{4,11,12,38,43}$ Elevated levels of pro-inflammatory cytokines involved in the pathogenesis of a variety of acute and chronic inflammatory diseases have been implicated in the endothelial and epithelial barrier dysfunction through cytoskeletal re-

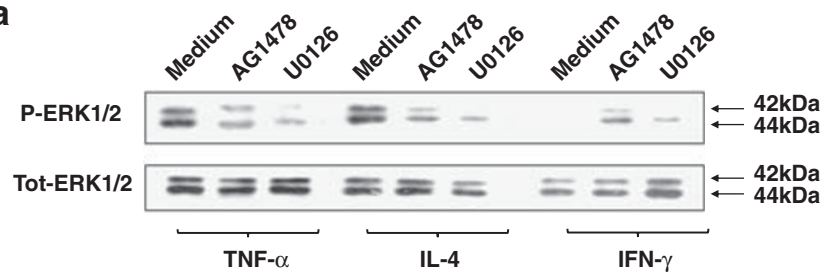

b

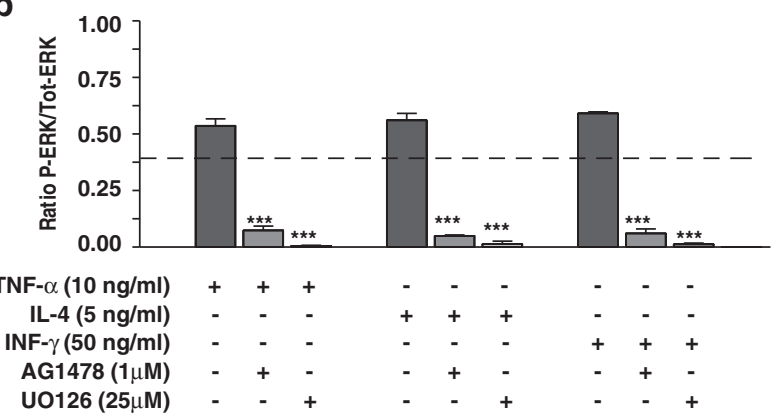

Figure 6 Detection by western blotting of the effect of epidermal growth factor receptor (EGFR) tyrosine kinase inhibitor (AG1478) and mitogenactivated protein kinase kinase (MEK) inhibitor (U0126) on cytokine-induced extracellular signal-regulated kinases (ERK)1/2 phosphorylation. (a) Representative western blots for total (Tot-ERK1/2) and phosphorylated ERK1/2 (P-ERK1/2) levels in Calu-3 cultured with medium alone (Medium) or exposed to TNF- $\alpha$, IL-4 and INF- $\gamma$ for $5 \mathrm{~min}$, in the presence of AG1478, $1 \mu \mathrm{M}$ or U0126, $25 \mu \mathrm{M}$. (b) Densitometric analysis of P-ERK1/2 data, normalized to Tot-ERK $1 / 2$ and plotted as means \pm s.e.m.. Dotted line represents P-ERK/ Tot-ERK ratio under basal conditions (Control). The results shown are representative of five independent experiments. ${ }^{* *} P<0.001$ versus cells exposed to cytokines in cultures without AG1478 or U0126.

arrangement in a variety of tissues. ${ }^{3,4,11-17,44-46}$ However, variable effects of these cytokines on the TJ cytoplasmic plaque protein $\mathrm{ZO}-1$ and $\mathrm{TJ}$ structure and on epithelial permeability have been described in the few studies performed on airway epithelial cells. Evaluating 16HBE14o-cells by transmission electron microscopy, no effect of IL-4, IL-8, IL-13 or IFN- $\gamma$ on ZO-1 expression was reported, ${ }^{16}$ whereas the combination of IL-4 and IFN- $\gamma$ appeared to be able to induce gaps in TJs. ${ }^{16}$ Exposure of Calu-3 cells to IL-4, IL-13 and IFN- $\gamma$ gave contrasting results, with dissociation between TJ structure and function. ${ }^{17}$ Indeed, analysis of the TJ-associated proteins by immunoblotting showed that IL-4 and IL-13 significantly reduced ZO-1 expression and modestly decreased occludin, whereas IFN- $\gamma$ downregulated ZO-1 and occludin expression to almost undetectable levels. ${ }^{17}$ Surprisingly, whereas IL-4 and IL-13 treatment resulted in a $70-75 \%$ decrease in barrier function at 24, 48 and $72 \mathrm{~h}$ incubation, in the presence of IFN$\gamma$ no changes were observed at 24 and $48 \mathrm{~h}$ incubation followed by a threefold enhancement at $72 \mathrm{~h} .{ }^{17}$ No adequate explanation was provided by the authors to account for the paradoxical effect of IFN- $\gamma$ on barrier function and ZO-1 expression. ${ }^{17}$

Using Calu-3 cells, we also found a more remarkable reduction of ZO-1 than of occludin expression in response to TNF- $\alpha$, IL- 4 and IFN- $\gamma$, and a more remarkable occludin and ZO- 1 spatial dislocation in response to IFN- $\gamma$ than to TNF- $\alpha$. 

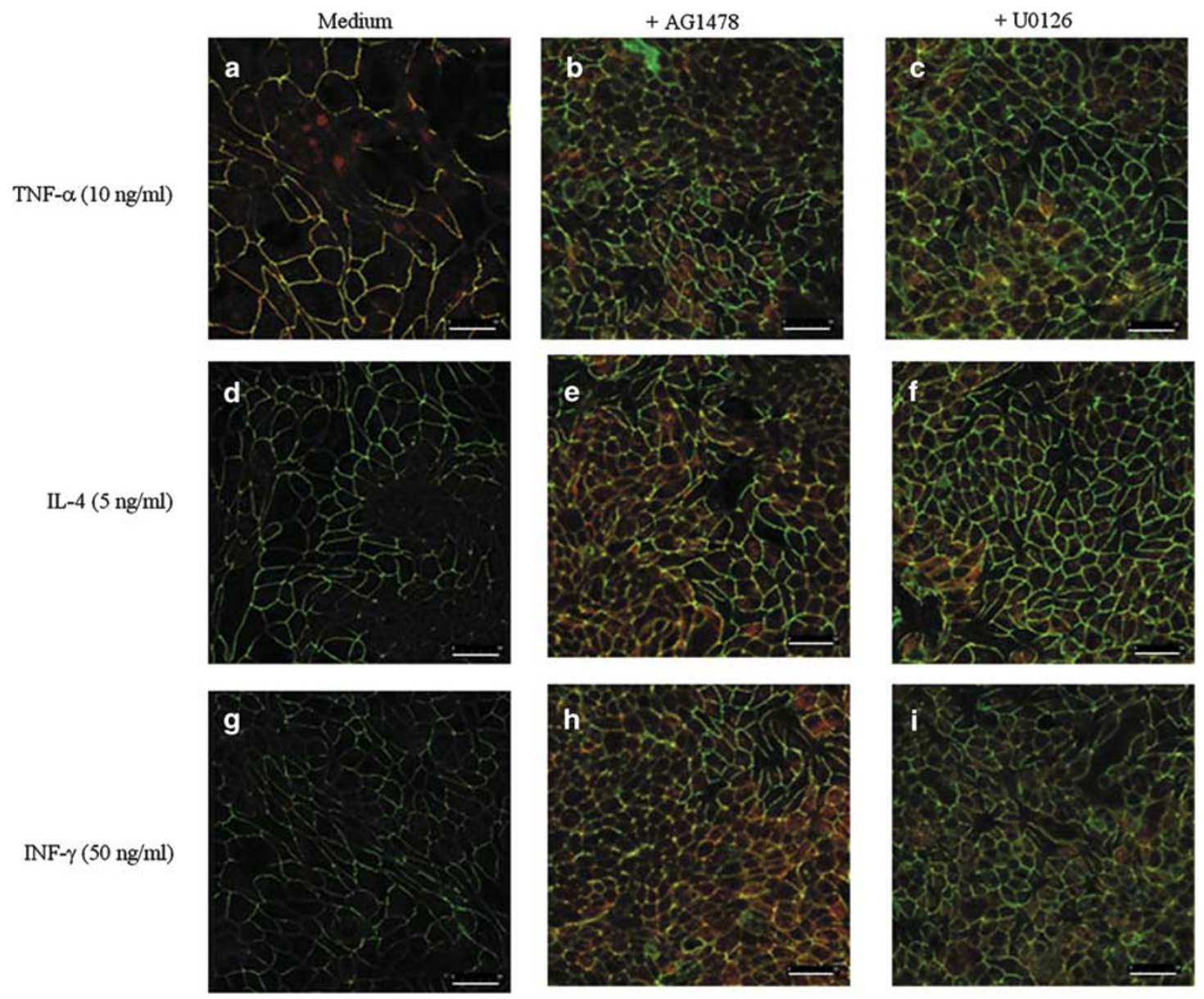

Figure 7 Effects of epidermal growth factor receptor (EGFR) and extracellular signal-regulated kinases (ERK)1/2 inhibitors on tight junction (TJ) disassembly induced by 48-h exposure to cytokines. Representative merged photomicrographs obtained by confocal microscopy after immunofluorescence staining, showing the inhibitory effect of pre-exposure of Calu-3 cells to AG1478 or UO126 on zonula occludens (ZO)-1 and occludin junctional disruption induced by TNF- $\alpha(\mathbf{a}-\mathbf{c})$, IL-4 $(\mathbf{d}-\mathbf{f})$ and INF- $\gamma(\mathbf{g}-\mathbf{i})$. Original magnification $\times 63$. The results shown are representative of the results obtained in four independent experiments. Scale bar: $50 \mu \mathrm{m}$.

TJ assembly disruption was associated with modification of the epithelial barrier integrity. In fact, the specific different concentrations of TNF- $\alpha$, IL- 4 or INF- $\gamma$ used to exanimate the effect on TJ protein reduction demonstrated a significant different decrease in TER in response to all three cytokines. In agreement with the results of the present paper, treatment of mouse tracheal epithelial cell monolayers with TNF- $\alpha$, IFN- $\gamma$ and IL- $1 \beta$ resulted in a markedly decreased TER after $24 \mathrm{~h} .{ }^{19}$ However, we also found a dissociation between the degree of cytokine-induced TJ disruption (highest for IFN- $\gamma$ ) and TER decrease (highest for IL-4), unexpected because of the importance ascribed to ZO-1 and occludin in the regulation of TJ function. In a different experimental model, exposure of primary human airway epithelial cells (16HBE14o-) to the nitric oxide donor (DETA-NO) resulted in an altered distribution and expression of TJ proteins (claudin-1 and occludin), not associated with significant barrier disruption. ${ }^{19}$

One explanation may be the redundancy of function provided by claudins, a new family of transmembrane pro- teins that also localize to TJs. ${ }^{47}$ There are $\geq 20$ known claudins, several of which interact with many of the same proteins as occludin. ${ }^{47}$ The existence of proteins with functional redundancy to occludin has been inferred from studies with occludin-deficient knockout mice that retain the ability to form morphologically TJs possessing normal barrier function. ${ }^{39}$ This occludin-like activity is mediated by the claudins. In fact, it is becoming apparent that claudins are the major transmembrane components of TJs and that the permeability properties of an epithelium are the product of claudin composition in these mutant animals. ${ }^{48}$ However, further detailed analyses of the functions of claudin at the cellular level are required for a better understanding of the molecular mechanism behind the epithelial barrier integrity.

Cell culture conditions may also be critical. In an airliquid interface co-culture system of human lung epithelial cells and of primary human pulmonary microvascular endothelial cells, it was shown that responses to several cytokines, activation of transcription factors and TNF reduction 


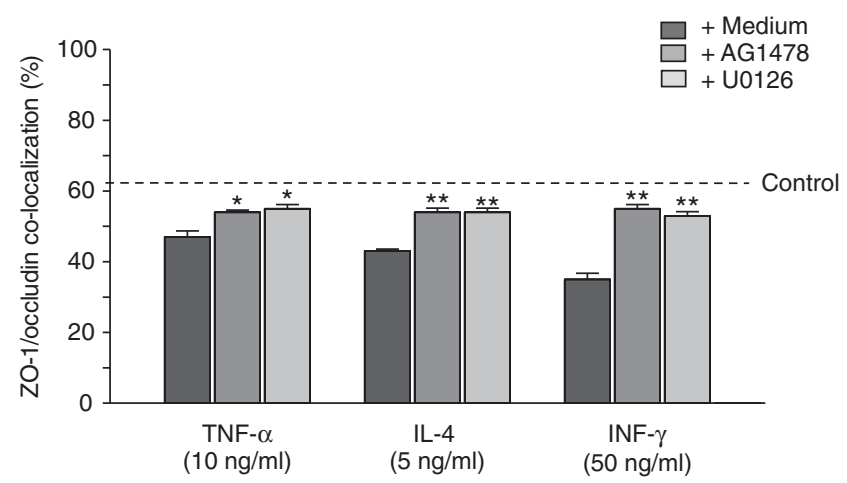

Figure 8 Quantitative evaluation of the activity epidermal growth factor receptor (EGFR) and extracellular signal-regulated kinases (ERK)1/2 inhibitors on tight junction (TJ) disassembly induced by exposure to cytokines. The percentage of zonula occludens (ZO)-1/occludin colocalization in Calu- 3 cells is expressed on the ordinate and the various culture conditions on the abscissa. Dotted line represents ZO-1/occludin colocalization under basal culture condition (Control). Data reported are representative of the results obtained in four independent experiments and are expressed as mean \pm s.e.m.. ${ }^{\star} P<0.05$ and ${ }^{* *} P<0.01$ versus cells exposed to cytokines in cultures without AG1478 or UO126.

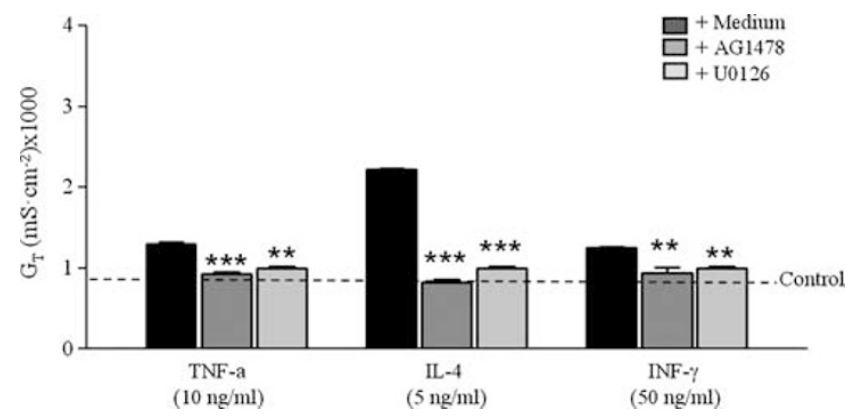

Figure 9 The effects of epidermal growth factor receptor (EGFR) and extracellular signal-regulated kinases (ERK) $1 / 2$ inhibitors on cytokineinduced changes in total conductance $\left(G_{T}\right)$. Dotted line represents total conductance $\left(G_{T}\right)$ under basal culture condition (Control). Data reported are representative of the results obtained in four independent experiments and are expressed as mean \pm s.e.m.. ${ }^{* * P}<0.01$ and ${ }^{* * *} P<0.001$ versus cells exposed to cytokines in cultures without AG1478 $(1 \mu \mathrm{M})$ or U0126 $(25 \mu \mathrm{M})$.

of TER values require basolateral mediator application, a condition favoring in vivo exposure to mediators released by immunoeffector cells in the airway walls. ${ }^{21,22}$ Epithelial permeability regulation is regulated by complex mechanisms, with a wide array of growth factors, cytokines, drugs and hormones exerting opposite effects on TJ organization and barrier function. ${ }^{1,2,21,22}$ Glucocorticoids, prolactin and unsaturated fatty acids, all enhance TJ barrier partially by increasing the expression of TJs whereas cytokines, oxidants and growth factors, as also reported here, increase permeability and mediate ZO-1 and/or occludin disruption in endothelial and epithelial cells. ${ }^{2,13,14}$ TJ permeability may be regulated directly through the modification of $\mathrm{TJ}$ proteins, but also indirectly through effects on the cytoskeleton, with the involvement of a variety of intracellular pathways, depending on the stimulus and on the cell type. ${ }^{2,20} \mathrm{TJ}$ integrity in different epithelia is regulated by G-proteins, protein kinase C, cSrc, phosphatidylinositol 3-kinase (PI3K), phospholipase $\mathrm{C} \gamma$ and protein phosphatase $2 \mathrm{~A} \cdot{ }^{2,49-51}$ Evidence suggests that these signaling activities may affect $\mathrm{TJs}$ by inducing phosphorylation and regulation of protein-protein interactions. ${ }^{50}$ In bronchial epithelial cells, various cellular processes are regulated by epidermal growth factor-like factors and the EGFR signaling pathways. ${ }^{12,23,24,27}$ EGFR activation turns on the MAPK/ERK1/2 pathway and mediates the promotion of cell proliferation, growth and differentiation. ${ }^{12,51,52}$ Various other external stimuli, including pro-inflammatory cytokines, that have the potential to disrupt epithelial junctions are known to activate the MAPK/ERK1/2 pathway, and previous studies have implicated ERK1/2 in disassembly of epithelial junctions at corneal level by phorbol 12-myristate 13-acetate ${ }^{53}$ at renal level by cyclosporine $\mathrm{A}^{54}$ and at airway level by cigarette smoke condensate. ${ }^{12}$ This has been confirmed in the present study where we showed on a bronchial epithelial cell line that MAPK/ERK pathway has a crucial role on protection of cytokines-induced TJ disruption and function.

\section{ACKNOWLEDGEMENTS}

Petecchia $L$ and Sabatini $F$ designed experiments, performed all the laboratory work, analyzed and interpreted data, generated figures and wrote the manuscript. Usai $C$ assisted with fluorimetric determination of the intracellular $\mathrm{Ca}^{2+}$ concentration. Caci $\mathrm{E}$ assisted with analysis of transepithelial electrical resistance. Varesio $L$ assisted with writing and participated in discussions. Rossi GA provided crucial ideas, designed experiments, analyzed and interpreted data, supervised the study, and cowrote the manuscript. This study was supported by Grant 'Ricerca Corrente' from Italian Ministry of Health, Rome-Italy. The work was performed in the Pulmonary Disease Unit, G.Gaslini Institute, Genoa, Italy.

\section{DISCLOSURE/CONFLICT OF INTEREST}

The authors declare no conflict of interest.

1. Robbins R, Rennard S. Biology of airway epithelial cells. In: Crystal RG, West JB, Barnes PJ (eds). The Lung: Scientific Foundations. LippincottRaven: Philadelphia, PA, 1997.

2. Harhaj N, Antonetti D. Regulation of tight junctions and loss of barrier function in pathophysiology. Int J Biochem Cell Biol 2004;36: 1206-1237.

3. Bruewer $\mathrm{M}$, Utech $\mathrm{M}$, Ivanov $\mathrm{Al}$, et al. Interferon-gamma induces internalization of epithelial tight junction proteins via a macropinocytosis-like process. FASEB J 2005;19:923-933.

4. Basuroy S, Seth A, Elias B, et al. MAPK interacts with occludin and mediates EGF-induced prevention of tight junction disruption by hydrogen peroxide. Biochem J 2006;393:69-77.

5. Fanning $A S$, Jameson $B J$, Jesaitis $L A$, et al. The tight junction protein ZO-1 establishes a link between the transmembrane protein occludin and the actin cytoskeleton. J Biol Chem 1998;273:29745-29753.

6. Furuse $M$, Itoh $M$, Hirase $T$, et al. Direct association of occludin with ZO1 and its possible involvement in the localization of occludin at tight junctions. J Cell Biol 1994;127(6 Pt 1):1617-1626.

7. Furuse $M$, Hirase $T$, Itoh $M$, et al. Occludin: a novel integral membrane protein localizing at tight junctions. J Cell Biol 1993;123(6 Pt 2):1777-1788.

8. Ando-Akatsuka $\mathrm{Y}$, Yonemura $\mathrm{S}$, Itoh $\mathrm{M}$, et al. Differential behavior of $\mathrm{E}-$ cadherin and occludin in their colocalization with ZO-1 during the establishment of epithelial cell polarity. J Cell Physiol 1999;179:115-125. 
9. Howarth $\mathrm{AG}$, Singer $\mathrm{KL}$, Stevenson BR. Analysis of the distribution and phosphorylation state of ZO-1 in MDCK and nonepithelial cells. J Membrane Biol 1994;137:261-270.

10. Belgiovine C, Chiodi I, Mondello C. Relocalization of cell adhesion molecules during neoplastic transformation of human fibroblasts. Int J Oncol 2011;39:1199-1204.

11. Turner JR. Molecular basis of epithelial barrier regulation: from basic mechanisms to clinical application. Am J Pathol 2006;169:1901-1909.

12. Petecchia L, Sabatini F, Varesio L, et al. Bronchial airway epithelial cell damage following exposure to cigarette smoke includes disassembly of tight junction components mediated by the ERK1/2 pathway. Chest 2009;135:1502-1512.

13. Sparrow MP, Omari TI, Mitchell HW. The epithelial barrier and airway responsiveness. Can J Physiol Pharmacol 1995;73:180-190.

14. Catalioto RM, Maggi CA, Giuliani S. Intestinal epithelial barrier dysfunction in disease and possible therapeutical interventions. Curr Med Chem 2011;18:398-426.

15. Capaldo CT, Nusrat A. Cytokine regulation of tight junctions. Biochim Biophys Acta 2009;1788:864-871.

16. Relova AJ, Shahana S, Makeeva N, et al. Effect of cytokines on ICAM-1 and ZO-1 expression on human airway epithelial cells. Cell Biol Int 2005;29:768-777.

17. Ahdieh $M$, Vandenbos $T$, Youakim $A$. Lung epithelial barrier function and wound healing are decreased by IL- 4 and IL-13 and enhanced by IFN-gamma. Am J Physiol Cell Physiol 2001;281:2029-2038.

18. Losa García JE, Rodríguez FM, Martín de Cabo MR, et al. Evaluation of inflammatory cytokine secretion by human alveolar macrophages. Mediators Inflamm 1999;8:43-51.

19. Olson N, Greul AK, Hristova M, et al. Nitric oxide and airway epithelial barrier function: regulation of tight junction proteins and epithelial permeability. Arch Biochem Biophys 2009;484:205-213.

20. Coyne CB, Vanhook MK, Gambling TM, et al. Regulation of airway tight junctions by proinflammatory cytokines. Mol Biol Cell 2002;13: 3218-3234.

21. Humlicek AL, Manzel LJ, Chin $\mathrm{CL}$, et al. Paracellular permeability restricts airway epithelial responses to selectively allow activation by mediators at the basolateral surface. J Immunol 2007;178:6395-6403.

22. Hermanns $\mathrm{Ml}$, Unger $\mathrm{RE}$, Kehe $\mathrm{K}$, et al. Lung epithelial cell lines in coculture with human pulmonary microvascular endothelial cells: development of an alveolo-capillary barrier in vitro. Lab Invest 2004;84: 736-752.

23. Pohl C, Hermanns Ml, Uboldi C, et al. Barrier functions and paracellular integrity in human cell culture models of the proximal respiratory unit. Eur J Pharm Biopharm 2009:72:339-349.

24. Agell N, Bachs O, Rocamora N, et al. Modulation of the Ras/Raf/MEK/ ERK pathway by Ca2+, and calmodulin. Cell Signal 2002;14:649-654.

25. Chang L, Karin M. Mammalian MAP kinase signalling cascades. Nature 2001;410:37-40.

26. Li D, Mrsny R. Oncogenic Raf-1 disrupts epithelial tight junctions via down-regulationof occludin. J Cell Biol 2000;148:791-800.

27. Kevil CG, Oshima T, Alexander B, et al. $\mathrm{H}(2) \mathrm{O}(2)$-mediated permeability: role of MAPK and occludin. Am J Physiol Cell Physiol 2000;279: C21-C30.

28. Fogh J, Fogh JM, Orfeo T. One hundred and twenty-seven cultured human tumor cell lines producing tumors in nude mice. J Natl Cancer Inst 1977;59:221-226.

29. Wan $\mathrm{H}$, Winton $\mathrm{HL}$, Soeller $\mathrm{C}$, et al. Tight junction properties of the immortalized human bronchial epithelial cell lines Calu-3 and 16HBE14o-. Eur Respir J 2000;15:1058-1068.

30. Olivera D, Boggs S, Beenhouwer C, et al. Cellular mechanisms of mainstream cigarette smoke-induced lung epithelial tight junction permeability changes in vitro. Inhal Toxicol 2007;19:13-22.

31. Ma TY, Boivin MA, Ye D, et al. Mechanism of TNF-\{alpha\} modulation of Caco-2 intestinal epithelial tight junction barrier: role of myosin light-chain kinase protein expression. Am J Physiol Gastrointest Liver Physiol 2005;288:422-430.
32. Manders EMM, Verbeek FJ, Aten JA. Measurement of co-localization of objects in dual-colour confocal images. J Microsc 1993;169:375-382.

33. Gonzalez RC, Wintz P. Digital Image Processing, 2nd edn. Addison Wesley Publication Company, Mass: USA, 1987.

34. Costes SV, Daelemans D, Cho EH, et al. Automatic and quantitative measurement of protein-protein colocalization in live cells. Biophys $J$ 2004;86:3993-4003.

35. Van Itallie C, Rahner C, Anderson JM. Regulated expression of claudin-4 decreases paracellular conductance through a selective decrease in sodium permeability. J Clin Invest 2001;107:1319-1327.

36. Kibangou IB, Bureau F, Allouche S, et al. Bouglé (Interactions between ethylenediaminetetraacetic acid (EDTA) and iron absorption pathways, in the Caco-2 model. Food Chem Toxicol 2008;46:3414-3416.

37. Vermeer PD, Denker J, Estin M, et al. MMP9 modulates tight junction integrity and cell viability in human airway Epithelia. Am J Physiol Lung Cell Mol Physiol 2009;296:L751-L762.

38. Bryant DM, Mostov KE. From cells to organs: building polarized tissue. Nat Rev Mol Cell Biol 2008;9:887-901.

39. Saitou M, Furuse M, Sasaki $H$, et al. Complex phenotype of mice lacking occludin, a component of tight junction strands molecular biology of the cell 2000;11:4131-4142.

40. Zegarra-Moran O, Monteverde M, Galietta $L$, et al. Functional analysis of mutations in the putative binding site for cystic fibrosis transmembrane conductance regulator potentiators. Interaction between activation and inhibition. J Biol Chem 2007;282:9098-9104.

41. Giepmans BN, van ljzendoorn SC. Epithelial cell-cell junctions and plasma membrane domains. Biochim Biophys Acta 2009;1788:820-831.

42. Bryant DM, Stow JL. The ins and outs of E-cadherin trafficking. Trends Cell Biol 2004;14:427-434 9.

43. Mankertz J, Schulzke JD. Altered permeability in inflammatory bowel disease: pathophysiology and clinical implications. Curr Opin Gastroenterol 2007;23:379-383.

44. Rogy MA, Coyle SM, Oldenburg HS, et al. Persistently elevated soluble tumor necrosis factor receptor and interleukin-1 receptor antagonist levels in critically ill patients. J Am Coll Surg 1994;178: 132-138.

45. Blum MS, Toninelli E, Anderson JM, et al. Cytoskeletal rearrangement mediates human microvascular endothelial tight junction modulation by cytokines. Am J Physiol Heart Circ Physiol 1997;273:286-294.

46. Oshima T, Laroux FS, Coe LL, et al. Interferon-gamma and interleukin10 reciprocally regulate endothelial junction integrity and barrier function. Microvasc Res 2001;61:130-143.

47. Tsukita S, Furuse M. Occludin and claudins in tight junction strands: leading or supporting players. Trends Cell Biol 1999;9:268-273.

48. Mitic LL, Van Itallie CM, Anderson JM. Molecular physiology and pathophysiology of tight junctions I. Tight junction structure and function: lessons from mutant animals and proteins. Am J Physiol Gastrointest Liver Physiol 2000;279:G250-G254 Review.

49. Ma TY, Iwamoto GK, Hoa NT, et al. TNF-alpha-induced increase in intestinal epithelial tight junction permeability requires NF-kappa B activation. Am J Physiol Gastrointest Liver Physiol 2004;286:367-376.

50. Aveleira CA, Lin CM, Abcouwer SF, et al. TNF- $\alpha$ signals through PKC $\zeta /$ $\mathrm{NF}-\kappa \mathrm{B}$ to alter the tight junction complex and increase retinal endothelial cell permeability. Diabetes 2010;59:2872-2882.

51. Scharl M, Paul G, Barrett $K E$, et al. AMP-activated protein kinase mediates the interferon-gamma-induced decrease in intestinal epithelial barrier function. J Biol Chem 2009;284:27952-27963.

52. Schramek H. MAP kinases: from intracellular signals to physiology and disease. News Physiol Sci 2002;17:62-67.

53. Wang Y, Zhang J, Yi XJ, et al. Activation of ERK1/2 MAP kinase pathway induces tight junction disruption in human corneal epithelial cells. Exp Eye Res 2004;78:125-136.

54. Martin-Martin N, Ryan G, McMorrow T, et al. Sirolimus and cyclosporine A alter barrier function in renal proximal tubular cells through stimulation of ERK1/2 signaling and claudin-1 expression. Am J Physiol Renal Physiol 2010;298:F672-F682. 